Artículo científico

Volumen 30(2):381-393. Mayo-agosto, 2019

e-ISSN 2215-3608, doi:10.15517/am.v30i2.32653

https://revistas.ucr.ac.cr/index.php/agromeso/index

\title{
Inhibición del crecimiento micelial de hongos asociados a antracnosis en name (Dioscorea alata) ${ }^{1}$
}

\section{Inhibition of mycelial growth of fungi associated with anthracnose in yam (Dioscorea alata)}

\author{
Cinthia Arce-Araya ${ }^{2}$, Ingrid Varela-Benavides ${ }^{3}$, Sergio Torres-Portuguez ${ }^{3}$
}

1 Recepción: 7 de marzo, 2018. Aceptación: 17 de octubre, 2018. Este trabajo formó parte de los resultados de la tesis de licenciatura "Efecto de fungicidas sobre el crecimiento micelial de aislamientos fúngicos asociados a antracnosis en ñame (Dioscorea alata) a nivel in vitro en Santa Clara, Costa Rica", enmarcada en el proyecto de investigación "Estudio de la antracnosis mediante herramientas biotecnológicas para el mejoramiento del sistema de producción en ñame (Dioscorea alata)", financiada por la Vicerrectoría de Investigación del Instituto Tecnológico de Costa Rica y la Fundación para el Fomento y Promoción de la Investigación y Transferencia de Tecnología Agropecuaria de Costa Rica (FITTACORI). Costa Rica.

2 Instituto Tecnológico de Costa Rica (ITCR), Sede San Carlos, Alajuela, Costa Rica. Tel: (506) 8642-1067. carce.2109@gmail.com

3 Instituto Tecnológico de Costa Rica (ITCR), Escuela de Agronomía, Sede San Carlos. Apartado 159-7050, Florencia, Alajuela, Costa Rica. Tel: (506) 2401-3047.invarela@itcr.ac.cr (https://orcid.org/0000-0003-1210-2664), storres@itcr.ac.cr

\section{Resumen}

Introducción. El ñame es afectado por gran cantidad de organismos como virus, bacterias y hongos, el más importante es Colletotrichum, causante de la antracnosis. Objetivo. El objetivo del presente trabajo fue determinar, por medio de un ensayo in vitro, la inhibición que ejercen fungicidas comerciales sobre el crecimiento micelial de siete aislamientos fúngicos que habían sido obtenidos de lesiones foliares en ñame. Materiales y métodos. El estudio se llevó a cabo en el Instituto Tecnológico de Costa Rica, Sede San Carlos, en el período comprendido entre octubre del 2016 y junio del 2017. Se evaluaron diez fungicidas (azoxistrobina, benomil, benzotiazol carbendazim, clorotalonil, mancozeb, mancozeb + oxicloruro de cobre, metil-tiofanato, propiconazol y propineb), con diversas concentraciones, sobre cinco aislamientos de Colletotrichum, uno de Bipolaris y uno de Mycoleptodiscus. Para ello, se realizaron dos ensayos, en el primero se analizó el crecimiento de los aislamientos en PDA con cinco diferentes concentraciones de cada fungicida. Los resultados obtenidos se utilizaron posteriormente en el segundo ensayo para calcular el porcentaje de inhibición del crecimiento micelial (PICM) de cada aislamiento. Resultados. Los resultados indican que, en las concentraciones utilizadas sobre los aislamientos fúngicos estudiados, ningún tratamiento alcanzó el $100 \%$ de inhibición. El propineb fue el fungicida más eficaz sobre el hongo Mycoleptodiscus sp., con un PICM de 64,51, el carbendazim tuvo un comportamiento similar sobre Colletotrichum truncatum con un PICM de 43,83 y el propiconazol lo tuvo sobre Colletotrichum gloeosporioides con un PICM de 49,79. La azoxistrobina no fue eficaz a ninguna concentración sobre ningún aislamiento. Conclusión. Los resultados obtenidos sugieren que los patógenos asociados a antracnosis en las plantaciones de ñame costarricenses podrían estar desarrollando resistencia a los fungicidas.

Palabras clave: fungicidas, hongos patógenos, Colletotrichum, inhibición del crecimiento micelial. 


\begin{abstract}
Introduction. Yam is a tuber affected by a large number of organisms such as viruses, bacteria and fungi, being Colletotrichum, which causes anthracnose, the most important. Objective. The objective of the present research was to determine, through an in vitro test, the inhibition exerted by commercial fungicides on the mycelial growth of seven fungal isolations that had been obtained from foliar lesions in yam. Materials and methods. The study was carried out at the Instituto Tecnológico de Costa Rica, in the period between October 2016 and June 2017. Ten fungicides -azoxystrobin, benomyl, benzotiazol, carbendazine, chlorothalonil, mancozeb, mancozeb + copper oxychloride, methylthiophanate, propiconazole, and propineb - were evaluated at different concentrations, on five isolates of Colletotrichum, one of Bipolaris and one of Mycoleptodiscus. With this purpose, two tests were performed; in the first, the growth of the isolates in PDA was analyzed in five different concentrations of each fungicide. The results obtained were subsequently used in the second test to calculate the percentage of inhibition of mycelial growth (PICM) of each isolation. Results. The results indicate that, in the concentrations used and with the fungal isolates studied, no treatment reached $100 \%$ inhibition. Propineb was the most effective fungicide on the fungus Mycoleptodiscus sp., with a PICM of 64.51, carbendazim had a similar behavior on Colletotrichum truncatum with a PICM of 43.83, and propiconazole had it on Colletotrichum gloeosporioides with a PICM of 49.79. Azoxystrobin was not effective at any concentration on any isolation. Conclusion. The results obtained suggest that the pathogens associated with anthracnose in Costa Rican yam plantations could be developing resistance to fungicides.
\end{abstract}

Keywords: fungicides, pathogenic fungi, Colletotrichum, inhibition of mycelial growth.

\title{
Introducción
}

El ñame (Dioscorea alata L., Dioscoreaceae) es un tubérculo comestible de gran importancia económica en regiones tropicales a nivel mundial, considerado un producto sustituto de la papa y de la yuca. Anteriormente, se consideraba un cultivo de subsistencia, sin embargo, desde los años noventa ha adquirido un papel más importante en la economía global, debido al aumento de su consumo y a que se ha extendido su uso en la industria farmacéutica, ya que la diosgenina que se extrae de sus tejidos, es un precursor en la síntesis de hormonas y cortisona (GonzálezVega, 2012).

En Costa Rica, el ñame representa cerca de un $1 \%$ de las exportaciones agrícolas, con una ganancia para el país cercana a los quince millones de dólares anuales. Según datos de la Promotora de Comercio Exterior de Costa Rica en el año 2017, entre enero y noviembre, se exportaron 13 861,7 t, lo que representó ingresos al país por 10,5 millones de dólares (PROCOMER, 2017).

El ñame se considera un cultivo generador de desarrollo, porque es producido en su mayoría por pequeños y medianos productores de áreas rurales (Medaglia, 2012). Esto se evidencia en el último censo nacional costarricense, cuyos resultados señalaron que en el 2014, había un total de 1076 productores de ñame y un promedio de cuatro hectáreas por productor, la mayor parte ubicados en las regiones Huetar Norte y Huetar Caribe del país (INEC, 2015).

Una de las principales limitantes en la producción del ñame es la antracnosis, atribuida al patógeno Colletotrichum (Ascomycota, Glomerellaceae, Sordariomycetes). La antracnosis del ñame se caracteriza por causar daño en el follaje, inicialmente aparecen pequeñas manchas marrones que luego se expanden provocando el oscurecimiento total de la lámina foliar. Cuando la enfermedad está muy avanzada la defoliación es completa. La antracnosis reduce la eficiencia fotosintética de las plantas de ñame afectando el rendimiento (Egesi et al., 2007).

Las pérdidas atribuidas a la antracnosis pueden alcanzar hasta un $80 \%$ en las plantaciones, y ha llevado a los productores a realizar aplicaciones desmedidas de fungicidas, con la consecuente presión de selección sobre cepas 
resistentes a los mismos (Abang et al., 2003). La generación de resistencia tiene como consecuencias el aumento de los costos de producción, la contaminación ambiental y en algunos casos el abandono total del cultivo (Alzate et al., 2009).

C. gloeosporioides ha mostrado la tendencia a presentar resistencia a los fungicidas en el mediano plazo, debido a que posee una extraordinaria capacidad de adaptación y variabilidad genética elevada. Desde el año 2003, estudios muestran la diversidad del género, y en particular en las cepas encontradas causando enfermedad en ñame. Los últimos análisis moleculares indican que la diversidad está bajo el nivel de especie (Weir et al., 2012; Suwannart et al., 2017).

En Costa Rica, las investigaciones sobre el manejo del cultivo de ñame han sido pocas, lo que ha limitado el establecimiento de programas de manejo efectivos. Para implementar medidas preventivas y curativas, resulta indispensable la identificación de los patógenos asociados a la antracnosis, su caracterización y determinación de su distribución, además del estudio de la susceptibilidad de tales patógenos a los productos utilizados para su control (Campo, 2011; Raj et al., 2013).

La carencia de información sobre la antracnosis en las plantaciones de ñame costarricenses condujo a investigadores del Instituto Tecnológico de Costa Rica, Sede San Carlos (ITCR-SSC) a proponer y ejecutar el proyecto de investigación: "Estudio de la antracnosis mediante herramientas biotecnológicas para el mejoramiento del sistema de producción en ñame (Dioscorea alata)". En el marco del proyecto, se realizaron encuestas a productores de ñame de las regiones Huetar Caribe y Huetar Norte, donde concluyó que el manejo y elección de los agroquímicos por parte de los productores para la prevención y la curación de la semilla vegetativa es deficiente. Entre los productores hubo mucha desinformación sobre el manejo de la enfermedad, lo que probablemente ha incidido en un aumento de la severidad de esta (Chaves, 2017; Sánchez, 2017). Además, como parte de las actividades del proyecto mencionado, se aislaron e identificaron hongos asociados a lesiones en el tejido vegetativo de ñame en fincas ubicadas en las regiones Huetar Caribe y Huetar Norte.

El objetivo del presente trabajo fue determinar, por medio de un ensayo in vitro, la inhibición que ejercen fungicidas comerciales sobre el crecimiento micelial de siete aislamientos fúngicos que habían sido obtenidos de lesiones foliares en ñame.

\section{Materiales y métodos}

\section{Localización del estudio}

El ensayo se realizó en el Laboratorio de Nematología del Instituto Tecnológico de Costa Rica, sede San Carlos, Alajuela, Costa Rica. Se inició en octubre del 2016 y finalizó en junio del 2017. Las coordenadas geográficas para la zona son $9^{\circ} 51^{\prime} 16,37^{\prime}$ ' $\mathrm{N}$ y $83^{\circ} 54^{\prime} 32,53$ ' O, se encuentra a $170 \mathrm{msnm}$, con una temperatura media anual de 26 ${ }^{\circ} \mathrm{C}$. La precipitación pluvial media es de $3500 \mathrm{~mm}$ anuales, con una estación lluviosa de mayo a diciembre, y una estación seca de enero a abril (IMN, 2008).

\section{Selección de aislamientos fúngicos y preparación de los cultivos}

Siete hongos que habían sido aislados de lesiones presentes en tejido vegetativo de ñame por Chaves (2017) y Sánchez (2017), se seleccionaron de un total de 150. Al realizar la selección se procuró que los aislamientos provinieran de diferentes sistemas productivos y de ambas regiones, Huetar Caribe y Huetar Norte. La identidad de cada aislamiento, la fecha y el lugar donde fueron colectados se observa en el Cuadro 1. 
Cuadro 1. Aislamientos fúngicos obtenidos de fincas productoras de name (Dioscorea alata), que fueron seleccionados para la realización de ensayos de susceptibilidad a fungicidas. Instituto Tecnológico de Costa Rica, sede San Carlos, Alajuela, Costa Rica. 2017.

Table 1. Fungal isolations obtained from yam (Dioscorea alata) producing landscapes, that were selected for carrying out fungicide susceptibility tests. Instituto Tecnológico de Costa Rica, San Carlos headquarter, Alajuela, Costa Rica. 2017.

\begin{tabular}{|c|c|c|c|c|}
\hline Aislamiento fúngico & Identificación & Fecha de colecta & Distrito de colecta & Ubicación geográfica \\
\hline 1 & Colletotrichum truncatum & $17 / 11 / 2015$ & $\begin{array}{c}\text { Santa Cecilia de La Cruz, } \\
\text { Upala }\end{array}$ & $11^{\circ} 03^{\prime} 45^{\prime \prime} \mathrm{N}-85^{\circ} 26^{\prime} 06^{\prime \prime} \mathrm{O}$ \\
\hline 2 & Colletotrichum sp. & 28/03/2015 & Acapulco de Los Chiles & $10^{\circ} 41^{\prime} 32^{\prime \prime} \mathrm{N}-84^{\circ} 36^{\prime} 36^{\prime \prime} \mathrm{O}$ \\
\hline 3 & Colletotrichum sp. & $15 / 03 / 2016$ & Los Laureles de Upala & $10^{\circ} 58^{\prime} 53^{\prime \prime} \mathrm{N}-85^{\circ} 18^{\prime} 07^{\prime \prime O}$ \\
\hline 4 & Colletotrichum sp. & $17 / 11 / 2015$ & $\begin{array}{l}\text { Asentamiento El Indio, } \\
\text { Pococí }\end{array}$ & $10^{\circ} 22^{\prime} 21^{\prime \prime} \mathrm{N}-83^{\circ} 51^{\prime} 14^{\prime \prime} \mathrm{O}$ \\
\hline 5 & $\begin{array}{l}\text { Colletotrichum } \\
\text { gloeosporioides }\end{array}$ & $17 / 11 / 2015$ & La Teresa de Pococí & $10^{\circ} 18^{\prime} 28^{\prime \prime} \mathrm{N}-83^{\circ} 48^{\prime} 36^{\prime \prime} \mathrm{O}$ \\
\hline 6 & Mycoleptodiscus sp. & $26 / 11 / 2015$ & Cartagena de Guácimo & $10^{\circ} 17^{\prime} 06^{\prime \prime} \mathrm{N}-83^{\circ} 37^{\prime} 57^{\prime \prime O}$ \\
\hline 7 & Bipolaris sp. & $26 / 11 / 2015$ & Cartagena de Guácimo & $10^{\circ} 17^{\prime} 06^{\prime \prime} \mathrm{N}-83^{\circ} 37^{\prime} 57^{\prime \prime O}$ \\
\hline
\end{tabular}

Los hongos se aislaron, reprodujeron y se realizaron cultivos monospóricos según la metodología descrita por Cañedo y Ames (2004). Los aislamientos se identificaron mediante amplificación y posterior secuenciación de las regiones ITS1-5.8S-ITS2 del gen rRNA ITS, se utilizaron los imprimadores ITS1/ITS4 (White et al., 1990). Los aislamientos se mantuvieron en platos Petri con PDA y en refrigeración, replicándose periódicamente para evitar la pérdida de sus características (Chaves, 2017; Sánchez, 2017).

Una vez seleccionados los siete aislamientos, se procedió a colocarlos en platos Petri de $9 \mathrm{~cm}$ de diámetro con medio PDA estéril para su crecimiento en oscuridad y a $25{ }^{\circ} \mathrm{C}$. Todas las evaluaciones se realizaron cuando los aislamientos tuvieron entre 15 y 20 días de haber sido colocados en el medio fresco para su crecimiento.

\section{Selección de fungicidas}

Diez fungicidas utilizados frecuentemente por productores nacionales de ñame para el control de antracnosis se seleccionaron para realizar la evaluación (Chaves, 2017; Sánchez, 2017). Además, se procuró que los productos a ensayar estuvieran recomendados para el combate de especies del género Colletotrichum, y que pertenecieran a diferentes grupos químicos, con diferente mecanismo de acción y movilidad en la planta (Cuadro 2).

\section{Primer ensayo: inhibición del crecimiento micelial por medio de la técnica del medio enmendado}

Se realizaron ensayos de inhibición por medio de la técnica del medio enmendado, para ello se utilizaron placas multipozos (cada placa tenía veinticuatro pozos de 1,7 cm de diámetro cada uno). En cada pozo se colocó $1 \mathrm{ml}$ de PDA al que se le agregó uno de los fungicidas. Para cada fungicida se evaluaron cinco concentraciones diferentes, además de un testigo sin fungicida, las concentraciones se eligieron y calcularon con base en la dosis comercial recomendada del producto. Se realizaron cuatro repeticiones por concentración para cada aislamiento (Cuadro 3).

Una vez preparado el medio enmendado, en cada pozo se colocaron $50 \mu 1$ de solución de esporas con una concentración de $1 \times 10^{5}$ conidios. $\mathrm{ml}^{-1}$, de uno de los aislamientos, considerando la metodología descrita por Trinidad-Cruz et al. (2013). Las placas se incubaron a $25^{\circ} \mathrm{C}$, en oscuridad, y se evaluó el crecimiento micelial o la ausencia del mismo a los tres y a los seis días después de la inoculación. 
Cuadro 2. Fungicidas seleccionados por grupo químico, modo de acción y presentación, para la evaluación in vitro de su eficacia en el control de aislamientos fúngicos asociados a antracnosis en ñame (Dioscorea alata). Instituto Tecnológico de Costa Rica, sede San Carlos, Alajuela, Costa Rica. 2017.

Table 2. Selected fungicides by chemical group, action mode, and presentation to evaluate the in vitro efficiency to control fungal isolations associated to anthracnosis in yam (Dioscorea alata). Instituto Tecnológico de Costa Rica, San Carlos headquarter, Alajuela, Costa Rica. 2017.

\begin{tabular}{|c|c|c|c|c|}
\hline Nombre común & Nombre químico & Grupo químico & Modo de acción & Presentación \\
\hline Azoxistrobina & $\begin{array}{l}\text { metil (e)-2\{2-[6-(2-cianofenoxi) pirimidin-4- } \\
\text { iloxi]fenil\}-3-metoxiacrilato }\end{array}$ & estrobilurina & sistémico & sólido \\
\hline Benomil & 1-benzimidazol-2-ilcarbamato de metilo & benzimidazol & sistémico & sólido \\
\hline Benzotiazol & 2-(tiocianatometiltio) benzotiazol & benzotiazol & sistémico & líquido \\
\hline Carbendazim & metil benzimidazol-2-ilcarbamato & benzimidazol & sistémico & líquido \\
\hline Clorotalonil & 2,4,5,6-tetracloro-isoftalonitrilo & $\begin{array}{l}\text { benzonitrilo } \\
\text { halogenado }\end{array}$ & contacto & líquido \\
\hline Mancozeb & $\begin{array}{l}\text { etilenbis (ditiocarbamato) de manganeso con } \\
\text { sal de zinc }\end{array}$ & ditiocarbamato & contacto & sólido \\
\hline $\begin{array}{l}\text { Mancozeb + oxicloruro } \\
\text { de cobre }\end{array}$ & $\begin{array}{l}\text { etilenbis (ditiocarbamato) de manganeso con } \\
\text { sal de zinc }+ \text { trihidróxido de cloruro de dicobre }\end{array}$ & ditiocarbamato & contacto & sólido \\
\hline Metil-tiofanato & dimetil 4,4'-(o-fenileno) bis(3-tioalofanato) & benzimidazol & sistémico & líquido \\
\hline Propiconazol & $\begin{array}{l}( \pm)-1-[2-(2,4-\text { diclorofenil })-4-p r o p i l-1,3- \\
\text { dioxolan-2-ilmetil]-1h-1,2,4-triazol }\end{array}$ & ditiocarbamato & contacto & líquido \\
\hline Propineb & $\begin{array}{l}\text { 1,2-propileno-bis (ditiocarbamato) de zinc } \\
\text { polimérico }\end{array}$ & triazol & contacto & sólido \\
\hline
\end{tabular}

Cuadro 3. Concentraciones de los fungicidas utilizados para la evaluación de su actividad sobre aislamientos fúngicos asociados a antracnosis en ñame (Dioscorea alata), por el método del medio enmendado. Instituto Tecnológico de Costa Rica, sede San Carlos, Alajuela, Costa Rica. 2017.

Table 3. Concentrations of fungicides used to evaluate their activity on fungal isolations associated with anthracnose in yam (Dioscorea alata), through the poisoned medium method. Instituto Tecnológico de Costa Rica, San Carlos headquarter, Alajuela, Costa Rica. 2017.

\begin{tabular}{lccccc}
\hline & \multicolumn{3}{c}{ Concentración $\left(\mathbf{m g ~ I A . m l}^{-1}\right)$} \\
\cline { 2 - 6 } & $\mathbf{1}$ & $\mathbf{2}$ & $\mathbf{3}$ & $\mathbf{4}$ & $\mathbf{5}$ \\
\hline Azoxistrobina & 0,20 & 0,40 & 0,45 & 0,50 & 0,60 \\
Benomil & 0,10 & 0,30 & 0,50 & 0,75 & 1,00 \\
Benzotiazol & 0,01 & 0,03 & 0,04 & 0,07 & 0,10 \\
Carbendazim & 0,05 & 0,15 & 0,50 & 1,00 & 1,50 \\
Clorotalonil & 0,10 & 0,50 & 2,00 & 3,50 & 5,00 \\
Mancozeb & 0,50 & 1,00 & 2,00 & 3,00 & 4,00 \\
Mancozeb + oxicloruro de cobre & 1,00 & 2,00 & 3,00 & 4,00 & 5,00 \\
Metil-tiofanato & 0,05 & 0,10 & 1,00 & 1,50 & 2,00 \\
Propiconazol & 0,05 & 0,10 & 0,15 & 0,20 & 0,25 \\
Propineb & 0,50 & 2,00 & 4,00 & 6,00 & 8,00 \\
\hline
\end{tabular}




\section{Segundo ensayo: determinación del porcentaje de inhibición del crecimiento micelial (PICM) mediante el método de difusión en agar}

Para la determinación del porcentaje de inhibición del crecimiento micelial (PICM) de cada aislamiento, se utilizó la técnica de difusión en agar (Bolouiri et al., 2016). Una unidad experimental se preparó inoculando 150

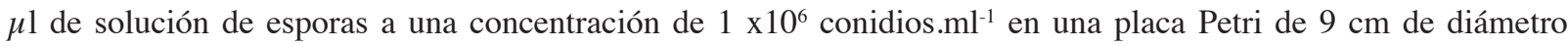
con PDA, la solución se distribuyó homogéneamente por toda la placa con ayuda de un asa bacteriológica estéril. Seguidamente, se colocó en el centro de la placa un disco de papel filtro de $6 \mathrm{~mm}$ de diámetro, impregnado con fungicida a una concentración determinada (Cuadro 4).

Cuadro 4. Concentraciones utilizadas para la evaluación de la inhibición del crecimiento micelial de aislamientos fúngicos asociados a antracnosis en ñame (Dioscorea alata), mediante el método de difusión en agar. Instituto Tecnológico de Costa Rica, sede San Carlos, Santa Clara, Alajuela, Costa Rica. 2017.

Table 4. Concentrations used for the evaluation of the inhibition of mycelial growth of fungal isolations associated with anthracnose in yam (Dioscorea alata), through the agar diffusion method. Instituto Tecnológico de Costa Rica, San Carlos headquarter, Alajuela, Costa Rica. 2017.

\begin{tabular}{lccc}
\hline & $\begin{array}{c}\text { Concentración 1 } \\
\left(\text { mg IA.ml }^{-1}\right)\end{array}$ & $\begin{array}{c}\text { Concentración 2 } \\
\left(\text { mg IA.ml }^{-1}\right)\end{array}$ & $\begin{array}{c}\text { Concentración comercial } \\
\left.\text { recomendada (mg IA.ml }^{-1}\right)\end{array}$ \\
\hline Benomil & 0,30 & 0,75 & $0,375-0,75$ \\
Benzotiazol & 0,03 & 0,07 & $0,075-0,15$ \\
Carbendazim & 0,15 & 1,00 & $0,13-0,88$ \\
Clorotalonil & 0,50 & 3,50 & $0,36-3,6$ \\
Mancozeb & 1,00 & 3,00 & $2,0-3,0$ \\
Mancozeb + oxicloruro de cobre & 2,00 & 4,00 & $3,05-4,6$ \\
Metil-tiofanato & 0,10 & 1,50 & $0,88-1,67$ \\
Propiconazol & 0,10 & 0,20 & $0,15-0,20$ \\
Propineb & 2,00 & 6,00 & $0,7-0,14$ \\
\hline
\end{tabular}

Durante el segundo ensayo no se evaluó la azoxistrobina, dado que no mostró actividad fungicida sobre ninguno de los aislamientos en el primer ensayo.

Los siete aislamientos se probaron con cada uno de los nueve fungicidas y con dos concentraciones por fungicida. Se prepararon tres repeticiones por tratamiento, además de dos testigos de cada aislamiento y por cada concentración. El testigo consistió en colocar en la placa Petri con el hongo un papel filtro impregnado con agua esterilizada.

El ensayo correspondió con un diseño experimental completamente al azar (DCA), asignando de manera aleatoria tres repeticiones de los tratamientos en cada una de las unidades experimentales. Seguidamente, las placas se incubaron a $25 \pm 2{ }^{\circ} \mathrm{C}$, en oscuridad durante seis días. Se realizaron dos evaluaciones del diámetro de crecimiento radial del hongo, la primera a los tres días después de establecido el ensayo y la segunda a los seis días, midiendo con la ayuda de un calibrador de Vernier.

Se estimó el porcentaje del crecimiento del hongo según la fórmula (1) señalada por Salazar et al. (2012).

$$
\% \text { Crecimiento del hongo }=\frac{\text { (diámetro en PDA sin fungicida }- \text { diámetro en PDA con fungicida })}{\text { diámetro en PDA sin fungicida }} \times 100
$$


El porcentaje de inhibición de crecimiento (PICM) se calculó tomando en cuenta que la inhibición es el inverso del crecimiento, y se calculó como sigue: \% de inhibición = 100 - \% de crecimiento del hongo.

\section{Análisis estadístico}

Se realizaron pruebas de modelos lineales mixtos generalizados (MLMG), a partir de una distribución no paramétrica con un nivel de significancia del 0,05. Además, para cada variable analizada, se realizó un ANOVA, complementado con la prueba de comparación múltiple (Di-Rienzo et al., 2002). Todos los análisis se realizaron en el programa InfoStat (InfoStat, 2011).

\section{Resultados}

\section{Primer ensayo: inhibición del crecimiento micelial por medio de la técnica del medio enmendado}

El propineb, el benzotiazol, el mancozeb y el mancozeb + oxicloruro de cobre, fueron eficaces en evitar el crecimiento micelial en todas las concentraciones evaluadas y sobre todos los aislamientos (Figura 1). Mientras que el metil-tiofanato, el benomil, el clorotalonil y el carbendazim, mostraron inhibición únicamente sobre algunos de los aislamientos y con las concentraciones más elevadas del producto (Figura 2).

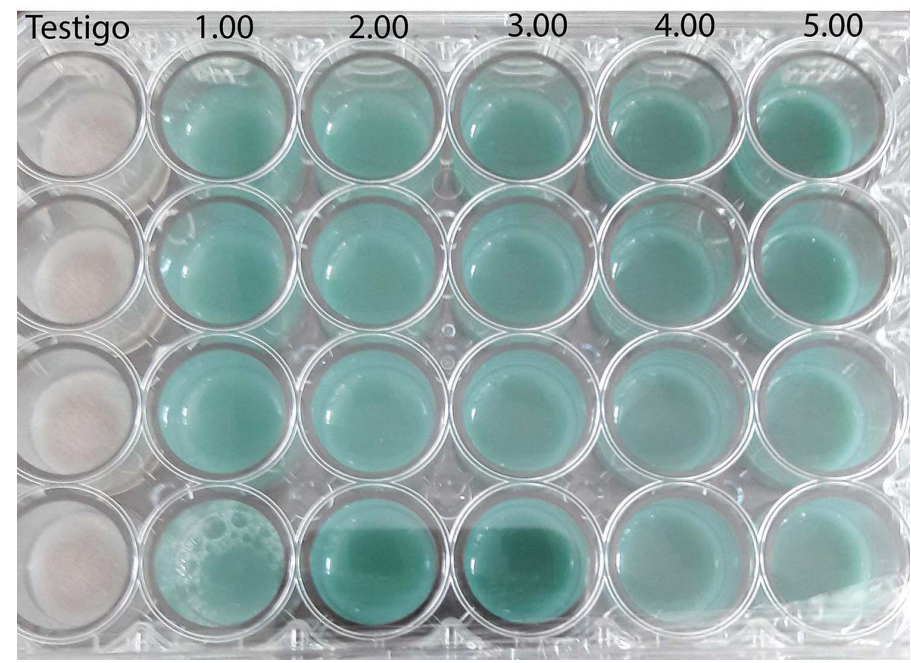

Figura 1. Resultados de la evaluación de la actividad fungicida de mancozeb + oxicloruro de cobre sobre Colletotrichum sp., aislado de name (Dioscorea alata). Cada columna de pozos corresponde a un tratamiento, y el valor indicado sobre la misma muestra la concentración (mg IA.ml ${ }^{-1}$ ) de fungicida evaluada en el mismo. En el tratamiento testigo se observa el crecimiento del hongo en todas las repeticiones, y en los demás tratamientos la ausencia de crecimiento. Instituto Tecnológico de Costa Rica, sede San Carlos, Alajuela, Costa Rica. 2017.

Figure 1. Results of the evaluation of the fungicidal activity of mancozeb + copper oxychloride on Colletotrichum sp., isolated from yam (Dioscorea alata). Each column of wells corresponds to a treatment, and the value indicated above each shows the concentration sample (mg mg IA.ml ${ }^{-1}$ ) of fungicide evaluated in it. In the control treatment it is observed the growth of the fungus in all repetitions, and in the other treatments the absence of growth. Instituto Tecnológico de Costa Rica, San Carlos headquarter, Alajuela, Costa Rica. 2017.

La azoxistrobina no controló el crecimiento de ningún aislamiento en ninguna de las concentraciones probadas (Figura 3). 


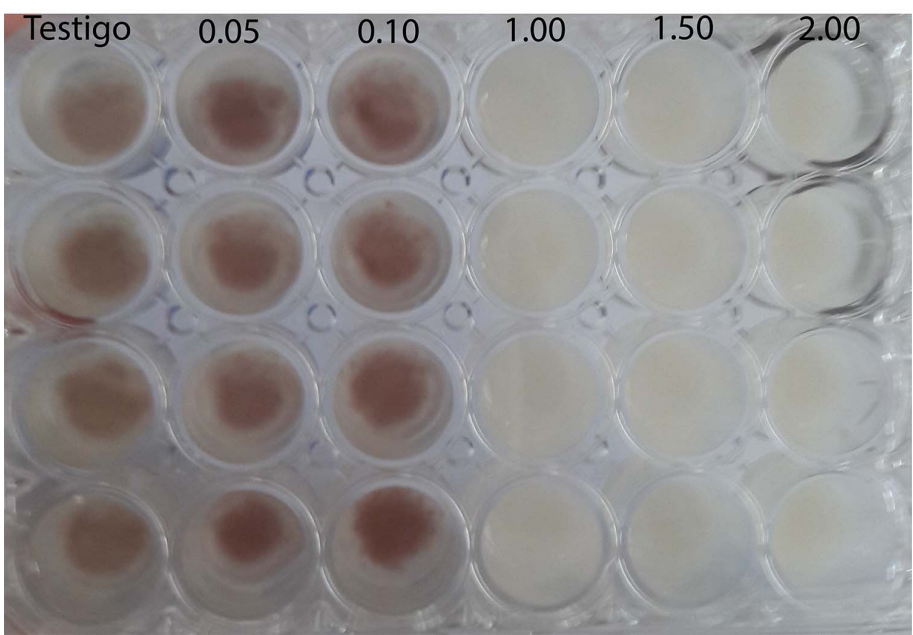

Figura 2. Resultados de la evaluación de la actividad fungicida de metil-tiofanato sobre Colletotrichum sp., aislado de ñame (Dioscorea alata). Cada columna de pozos corresponde a un tratamiento, y el valor indicado sobre la misma muestra la concentración (mg IA.ml-1) de fungicida evaluada en el mismo. En el testigo y tratamientos con baja concentración de fungicida se observa el crecimiento del hongo en todas las repeticiones, y en los tratamientos con mayor concentración de fungicida la ausencia de crecimiento. Instituto Tecnológico de Costa Rica, sede San Carlos, Alajuela, Costa Rica. 2017.

Figure 2. Results of the evaluation of the fungicide activity of methyl-thiophanate on Colletotrichum sp., isolated from yam (Dioscorea alata). Each column of wells corresponds to a treatment, and the value indicated above each shows the concentration (mg IA.ml ${ }^{-1}$ ) of fungicide evaluated in it. In the control and treatments with low concentration of fungicide fungus growth is observed in all the repetitions, and in the treatments with higher concentration of fungicide the absence of growth. Instituto Tecnológico de Costa Rica, San Carlos headquarter, Alajuela, Costa Rica. 2017.

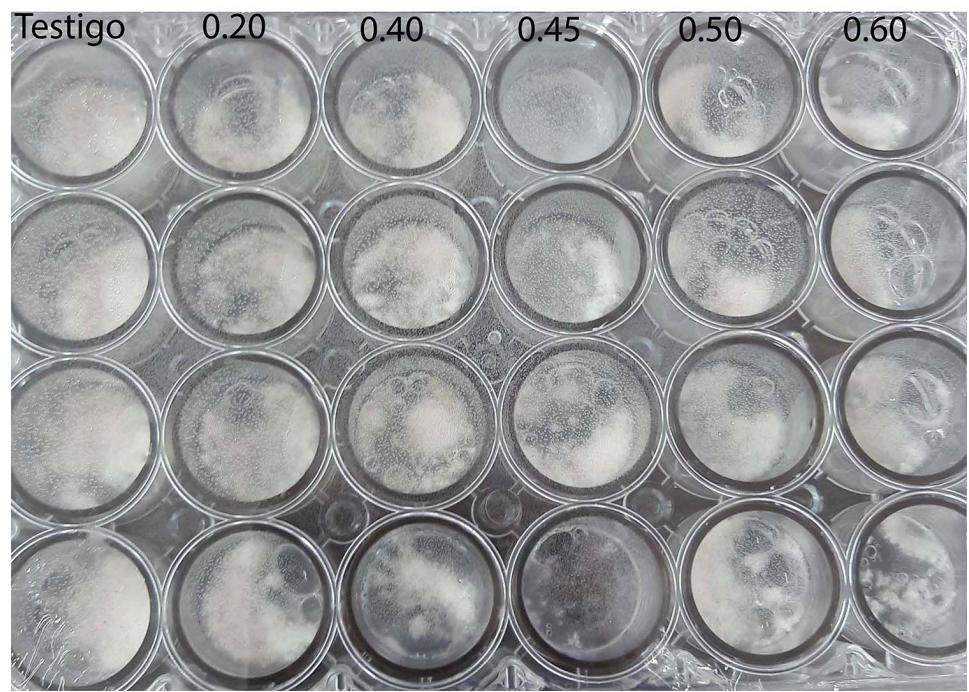

Figura 3. Resultados de la evaluación de la actividad fungicida de azoxystrobina sobre Colletotrichum truncatum. Cada columna de pozos corresponde a un tratamiento, y el valor indicado sobre la misma muestra la concentración (mg IA.ml ${ }^{-1}$ ) de fungicida evaluada en el mismo. En el tratamiento testigo y en los demás tratamientos se observa el crecimiento del hongo en todas las repeticiones. Instituto Tecnológico de Costa Rica, sede San Carlos, Alajuela, Costa Rica. 2017.

Figure 3. Results of the evaluation of the fungicidal activity of azoxystrobin on Colletotrichum truncatum. Each column of wells corresponds to a treatment, and the value indicated above each shows the concentration $\left(\mathrm{mg} \mathrm{IA.ml}^{-1}\right)$ of fungicide evaluated in it. In the control treatment and in the other treatments the growth of the fungus is observed in all the repetitions. Instituto Tecnológico de Costa Rica, San Carlos headquarter, Alajuela, Costa Rica. 2017. 


\section{Segundo ensayo: determinación del porcentaje de inhibición del crecimiento micelial (PICM) mediante el método de difusión en agar}

Los resultados obtenidos en las evaluaciones del PICM se muestran en el Cuadro 5. No se encontraron diferencias significativas $(p>0,05)$ entre los resultados obtenidos con las ecuaciones a los tres y seis días después de la inoculación, por lo que se presentan únicamente los resultados de la primera evaluación, que se realizó tres días después de establecido el ensayo.

Cuadro 5. Porcentaje de inhibición del crecimiento micelial (PICM) de fungicidas sobre aislamientos fúngicos obtenidos de plantas de ñame (Dioscorea alata) con síntomas de antracnosis. Instituto Tecnológico de Costa Rica, sede San Carlos, Santa Clara, Alajuela, Costa Rica. 2017.

Table 5. Percentage of inhibition of mycelial growth (PIMG) of fungicides on fungal isolations obtained from yam plants (Dioscorea alata) with symptoms of anthracnose. Instituto Tecnológico de Costa Rica, San Carlos headquarter, Alajuela, Costa Rica. 2017.

\begin{tabular}{|c|c|c|c|c|c|c|c|c|}
\hline \multirow[t]{2}{*}{ Fungicida } & \multirow{2}{*}{$\begin{array}{l}\text { Aislamiento } \\
\text { Concentra- } \\
\text { ción }(\mathbf{m g} \\
\left.\text { IA. } \mu \mathbf{l}^{-1}\right)\end{array}$} & $\begin{array}{c}\text { Colletotri- } \\
\text { chum trun- } \\
\text { catum } \\
\end{array}$ & $\begin{array}{l}\text { Colletotri- } \\
\text { chum sp. }\end{array}$ & $\begin{array}{l}\text { Colletotri- } \\
\text { chum sp. }\end{array}$ & $\begin{array}{l}\text { Colletotri- } \\
\text { chum sp. }\end{array}$ & $\begin{array}{c}\text { Colletotri- } \\
\text { chum gloeos- } \\
\text { porioides }\end{array}$ & $\begin{array}{l}\text { Mycolepto- } \\
\text { discus sp. }\end{array}$ & $\begin{array}{c}\text { Bipolaris } \\
\text { sp. }\end{array}$ \\
\hline & & 1 & 2 & 3 & 4 & 5 & 6 & 7 \\
\hline \multirow[t]{2}{*}{ Benomil } & 0,30 & $15,43 \mathrm{f}^{*}$ & $15,37 \mathrm{f}$ & $9,73 \mathrm{f}$ & $41,37 \mathrm{c}$ & $0,00 \mathrm{~g}$ & $52,12 \mathrm{~b}$ & $0,00 \mathrm{~g}$ \\
\hline & 0,75 & $12,07 \mathrm{f}$ & $11,79 \mathrm{f}$ & $4,50 \mathrm{~g}$ & $37,92 \mathrm{~d}$ & $0,00 \mathrm{~g}$ & $52,2 \mathrm{~b}$ & $0,00 \mathrm{~g}$ \\
\hline \multirow[t]{2}{*}{ Benzotiazol } & 0,03 & $15,76 \mathrm{f}$ & $13,07 \mathrm{f}$ & $14,81 \mathrm{f}$ & $28,90 \mathrm{~d}$ & $25,21 \mathrm{e}$ & $12,55 \mathrm{f}$ & $19,25 \mathrm{e}$ \\
\hline & 0,07 & $21,38 \mathrm{e}$ & $12,72 \mathrm{f}$ & $28,92 \mathrm{~d}$ & $20,43 \mathrm{e}$ & $35,96 \mathrm{~d}$ & $16,49 \mathrm{f}$ & $40,79 \mathrm{c}$ \\
\hline \multirow[t]{2}{*}{ Carbendazim } & 0,15 & $22,28 \mathrm{e}$ & $15,49 \mathrm{f}$ & $16,97 \mathrm{f}$ & $0,00 \mathrm{~g}$ & $0,00 \mathrm{~g}$ & $33,14 \mathrm{~d}$ & $0,00 \mathrm{~g}$ \\
\hline & 1,00 & $35,43 \mathrm{~d}$ & $27,64 \mathrm{e}$ & $28,82 \mathrm{~d}$ & $41,37 \mathrm{c}$ & $0,00 \mathrm{~g}$ & $24,35 \mathrm{e}$ & $0,00 \mathrm{~g}$ \\
\hline \multirow[t]{2}{*}{ Clorotalonil } & 0,50 & $15,50 \mathrm{f}$ & $5,08 \mathrm{~g}$ & $0,00 \mathrm{~g}$ & $7,22 \mathrm{~g}$ & $13,17 \mathrm{f}$ & $14,51 \mathrm{f}$ & $2,75 \mathrm{~g}$ \\
\hline & 3,50 & $15,05 \mathrm{f}$ & $6,30 \mathrm{~g}$ & $0,00 \mathrm{~g}$ & $22,31 \mathrm{e}$ & $8,36 \mathrm{~g}$ & $17,80 \mathrm{f}$ & $27,67 \mathrm{e}$ \\
\hline \multirow[t]{2}{*}{ Mancozeb } & 1,00 & $29,91 \mathrm{~d}$ & $4,19 \mathrm{~g}$ & $7,64 \mathrm{~g}$ & $8,30 \mathrm{~g}$ & $8,42 \mathrm{~g}$ & $8,43 \mathrm{~g}$ & $15,26 \mathrm{f}$ \\
\hline & 3,00 & $7,86 \mathrm{~g}$ & $7,56 \mathrm{~g}$ & $7,56 \mathrm{~g}$ & $13,78 \mathrm{f}$ & $9,8 \mathrm{f}$ & $10,71 \mathrm{f}$ & $14,59 \mathrm{f}$ \\
\hline \multirow{2}{*}{$\begin{array}{l}\text { Mancozeb + } \\
\text { oxicloruro de } \\
\text { cobre }\end{array}$} & 2,00 & $19,53 \mathrm{e}$ & $11,63 \mathrm{f}$ & $11,24 \mathrm{f}$ & 21,69 e & $9,63 \mathrm{f}$ & $13,45 \mathrm{f}$ & $7,06 \mathrm{~g}$ \\
\hline & 4,00 & $21,52 \mathrm{e}$ & $11,06 \mathrm{f}$ & $13,87 \mathrm{f}$ & $15,65 \mathrm{f}$ & $13,71 \mathrm{f}$ & $2,00 \mathrm{~g}$ & $11,06 \mathrm{f}$ \\
\hline \multirow[t]{2}{*}{ Metil-tiofanato } & 0,10 & $0,00 \mathrm{~g}$ & $0,00 \mathrm{~g}$ & $0,00 \mathrm{~g}$ & $0,00 \mathrm{~g}$ & $0,00 \mathrm{~g}$ & $0,00 \mathrm{~g}$ & $0,00 \mathrm{~g}$ \\
\hline & 1,50 & $0,00 \mathrm{~g}$ & $0,00 \mathrm{~g}$ & $0,00 \mathrm{~g}$ & $43,57 \mathrm{c}$ & $0,00 \mathrm{~g}$ & $0,00 \mathrm{~g}$ & $0,00 \mathrm{~g}$ \\
\hline \multirow[t]{2}{*}{ Propiconazol } & 0,10 & $0,00 \mathrm{~g}$ & $14,47 \mathrm{f}$ & $3,99 \mathrm{~g}$ & $1,25 \mathrm{~g}$ & $35,63 \mathrm{~d}$ & $16,55 \mathrm{f}$ & $26,71 \mathrm{e}$ \\
\hline & 0,20 & $0,00 \mathrm{~g}$ & $26,06 \mathrm{e}$ & $1,55 \mathrm{~g}$ & $7,33 \mathrm{~g}$ & $49,79 \mathrm{~b}$ & $11,86 \mathrm{f}$ & $29,18 \mathrm{~d}$ \\
\hline \multirow[t]{2}{*}{ Propineb } & 2,00 & $38,62 \mathrm{~d}$ & $20,00 \mathrm{e}$ & $23,84 \mathrm{e}$ & $51,92 \mathrm{~b}$ & $0,00 \mathrm{~g}$ & $64,51 \mathrm{a}$ & $0,00 \mathrm{~g}$ \\
\hline & 6,00 & $28,71 \mathrm{~d}$ & $23,74 \mathrm{e}$ & $25,54 \mathrm{e}$ & $58,71 \mathrm{a}$ & $8,42 \mathrm{~g}$ & $56,24 \mathrm{a}$ & $0,00 \mathrm{~g}$ \\
\hline
\end{tabular}

*Medias con una letra común en la misma columna no son significativamente diferentes $(p>0,05)$ según la prueba de comparación múltiple / Stocks with a common letter in the same column are not significantly different ( $>>0.05)$ according to the multiple comparison test.

Al comparar los resultados obtenidos durante las evaluaciones del primer y segundo ensayo, se observó que los resultados coincidieron en la mayoría de los casos, sin embargo, hubo excepciones: ni el benomil ni el carbendazim inhibieron el crecimiento de Mycoleptodiscus sp., durante el ensayo en medio enmendado; no obstante, los PICM obtenidos fueron similares o mayores a los mostrados sobre otros aislamientos. De la misma forma, el propineb resultó efectivo contra Bipolaris sp. en el medio enmendado, pero el PICM fue de 0. 


\section{Discusión}

Los porcentajes de inhibición del crecimiento micelial (PICM) obtenidos fueron bajos, si se comparan con los reportados en ensayos similares, por lo que podría interpretarse como una prueba de que los patógenos asociados a la antracnosis en las plantaciones de ñame costarricenses podrían estar desarrollando resistencia a los fungicidas (Gaviria-Hernández et al., 2013; Chacko y Gokulapalan, 2015).

Con respecto a otras pruebas de sensibilidad a fungicidas sobre Colletotrichum aislado de otros cultivos, se obtuvo PICM del $100 \%$ con propiconazol y $74 \%$ con carbendazim, sobre C. capsici aislado de chile (Chacko y Gokulapalan, 2015), estos porcentajes de inhibición fueron superiores a los obtenidos en la presente investigación, donde los mayores PICM obtenidos con propiconazol y carbendazim fueron de 49,8 \% sobre C. gleosporoides y $41 \%$ sobre el aislamiento 4 (Colletrotrichum sp.), respectivamente.

En esta investigación el metil-tiofanato tuvo un comportamiento similar a la azoxistrobina, ya que únicamente tuvo efecto inhibitorio en el crecimiento micelial de uno de los siete aislamientos, estos resultados difieren con lo reportado por Pérez-León et al. (2015) que determinaron que la mezcla de metil tiofanato + mancozeb proveyó un $100 \%$ de protección contra Colletotrichum a las hojas de Sansevieria. Además, las inconsistencias encontradas entre el primer y segundo ensayo pudieron deberse a la solubilidad de algunos de estos productos y su capacidad de difusión, efecto que ya ha sido mencionado por otros autores como un factor a tomar en cuenta al realizar diferentes pruebas de sensibilidad a los plaguicidas (Alzate et al., 2009; Gaviria-Hernández et al., 2013).

La resistencia de Colletotrichum y otros patógenos fúngicos a los fungicidas, ya ha sido reportado para otros sistemas de producción y en otras latitudes, y se ha atribuido al uso desmedido de estos productos. Distintos autores han reportado la tendencia de $C$. gloeosporioides en específico a presentar resistencia a los fungicidas en el mediano plazo, debido a que posee una extraordinaria capacidad de adaptación y variabilidad genética elevada (GutiérrezAlonso et al., 2003).

Aunado a la capacidad de adaptación de hongos como C. gloeosporioides, moléculas como la azoxistrobina y los benzimidazoles, son considerados de alto riesgo de resistencia cuando se utilizan periódicamente (GutiérrezAlonso y Gutiérrez-Alonso, 2003; Gutiérrez-Alonso et al., 2003).

Algunos aislamientos de Colletrotrichum spp. aislados de guayaba, papaya, nuez y pimienta, melocotón y arándanos, han presentado resistencia a la azoxistrobina (Gutiérrez-Alonso y Gutiérrez-Alonso 2003; Hu et al. 2015; Torres-Calzada et al., 2015). Además, de la resistencia de Colletotrichum hacia los benzimidazoles, grupo al cual pertenece el benomil y el carbendazim (Tavares y de-Souza, 2005).

Los benzimidazoles han sido los fungicidas más utilizados para el control de los patógenos asociados a la antracnosis desde la década de los noventa (Gutiérrez-Alonso y Gutiérrez-Alonso, 2003). Estas moléculas actúan por inhibición de la división nuclear a través de la mitosis por unión a las subunidades de la tubulina b (TUB2), en donde la resistencia a ellos se ha atribuido a mutaciones puntuales en el ADN, resultando en intercambios de aminoácidos en la proteína b-tubulina. La resistencia a los fungicidas metil-benzimidazol carbamatos se ha detectado en muchas especies de hongos (Ma y Michailides, 2005; Hu et al., 2015; Torres-Calzada et al., 2015).

En el caso de la azoxistrobina algunos informes han descrito que esa resistencia se debe a una mutación puntual en el codón 143 en el gen cyt b, que da lugar a una sustitución de la glicina por alanina (G143A), y confiere resistencia a las estrobilurinas (Heaney et al., 2000; Sierotzki et al., 2007).

En las plantaciones de ñame costarricenses, fungicidas como el benomil y la azoxistrobina, que en esta investigación resultó ineficaz para controlar todos los patógenos ensayados, están entre los fungicidas más utilizados para el control de la antracnosis. La azoxistrobina es el tratamiento de elección para el control de antracnosis en plantaciones de ñame, y los agricultores realizan varias aplicaciones durante el ciclo de cultivo (Chaves, 2017; Sánchez, 2017). 
En cuanto al mancozeb, aunque fue uno de los fungicidas que resultó eficaz en todos los aislamientos, tampoco alcanzó los porcentajes de inhibición contra Colletotrichum capsici reportados en la literatura (70 \%) (Chacko y Gokulapalan, 2015). Además, este producto se utiliza para el control de antracnosis por productores de las regiones Huetar Norte y Huetar Caribe en un 28,6 \% y 7 \%, respectivamente (Chaves, 2017; Sánchez, 2017), lo que podría explicar su baja eficacia.

Otro resultado que debe considerarse es que los mayores PICM se obtuvieron sobre los aislamientos de la región Huetar Caribe, mientras que en la región Huetar Norte el mayor PICM fue de 38 sobre C. truncatum. Tal diferencia podría deberse a que, en la región Huetar Norte, a diferencia de la Huetar Caribe, las plantaciones de ñame eran, en general, más numerosas y grandes, además de que la planta se sembraba directamente sobre el suelo, probablemente favoreciendo las condiciones climáticas que hacen que proliferen los patógenos estudiados.

En esta investigación fueron evidentes las diferencias en los PICM que ejercieron los diferentes fungicidas sobre los aislamientos (Cuadro 5). Tales resultados sugieren que la eficacia del control químico de antracnosis en las plantaciones de ñame costarricenses dependerá del patógeno causante de la enfermedad y su susceptibilidad al fungicida aplicado, por ejemplo, el benomil y el propineb mostraron un PICM sobre el $50 \%$ aplicado al aislamiento de Mycoleptodiscus sp., sin embargo, el control sobre el aislamiento de Colletotrichum gloeosporioides fue muy bajo, siendo este último uno de los aislamientos que presentó menor sensibilidad a los fungicidas probados, únicamente con el benzotiazol y el propiconazol se lograron obtener PICM sobre 20. Resultados similares se observaron sobre el asilamiento 7 (Bipolaris sp.). Tales resultados apoyan lo sugerido por Méndez et al. (2013), quienes apuntaron que una sola especie de Colletotrichum puede infectar múltiples huéspedes y, además, un solo huésped puede ser infectado por varias especies, siendo que las diferencias en los aislamientos patogénicos entre dos o más regiones probablemente reflejan las diferencias a escala genética en el germoplasma usado y las prácticas agrícolas desarrolladas en cada región.

\section{Conclusiones}

Ninguno de los fungicidas evaluados alcanzó el $100 \%$ de inhibición del crecimiento micelial sobre los aislamientos, además la eficacia de cada uno fue diferente y dependiente del aislamiento sobre el cual fue evaluado. Los resultados evidenciaron el desarrollo de resistencia diferencial de los patógenos asociados a la antracnosis del ñame, y la necesidad de realizar más investigación dirigida a mejorar el manejo integrado de esta enfermedad.

\section{Literatura citada}

Abang, M.M., S. Winter, H.D. Mignouna, K.R. Green, and R. Asiedu. 2003. Molecular taxonomic, epidemiological and population genetic approaches to understanding yam anthracnose disease. Afr. J. Biotechnol. 2:486-496. doi:10.5897/ AJB2003.000-1098

Alzate, D.A., G.I. Mier, L. Afanador, D.L. Durango, y C.M. García. 2009. Evaluación de la fitotoxicidad y la actividad antifúngica contra Colletotrichum acutatum de los aceites esenciales de tomillo (Thymus vulgaris), limoncillo (Cymbopogon citratus), y sus componentes mayoritarios. Vitae 16(1):116-125.

Balouiri, M., M. Sadiki, and S.K. Ibnsouda. 2016. Methods for in vitro evaluating antimicrobial activity: A review. J. Pharm. Anal. 6(2):71-79. doi:10.1016/j.jpha.2015.11.005

Campo, R.O. 2011. Manejo integrado de la antracnosis (Colletotrichum spp.) en ñame (Dioscorea alata) mediante el uso de alternativas para reducir el inóculo primario, la dispersión y el establecimiento del patógeno. Informe final de proyecto. Universidad de Córdoba, COL. doi:10.13140/RG.2.1.2576.9846

Agron. Mesoam. 30(2):381-393, mayo-agosto, 2019 
Cañedo, V., y T. Ames. 2004. Manual de laboratorio para el manejo de hongos entomopatógenos. Centro Internacional de la Papa, Lima, PER.

Chacko, S.T., and C. Gokulapalan. 2015. In vitro study of fungicides and biocontrol agents against Colletotrichum capsici causing anthracnose of chilli (Capsicum annuumm L.). Int. J. Appl. Pure Sci. Agric. 1(5):93-98.

Chaves, S. 2017. Identificación de patógenos asociados a antracnosis en el cultivo de ñame (Dioscorea alata) en la región Huetar Norte de Costa Rica. Tesis Bach., Instituto Tecnológico de Costa Rica, Santa Clara, CRI.

Di-Rienzo, J.A., A.W. Guzmán, and F. Casanoves. 2002. A multiple-comparisons method based on the distribution of the root node distance of a binary tree. J. Agric. Biol. Environ. Stat. 7:129-142. doi:10.1198/10857110260141193

Egesi, C.N., T. J. Onyeka, and R. Asiedu. 2007. Severity of anthracnose and virus diseases of water yam (Dioscorea alata L.) in Nigeria I: Effects of yam genotype and date of planting. Crop Prot. 26:1259-1265. doi:10.1016/j.cropro.2006.10.025

Gaviria-Hernández, V., L.F. Patiño-Hoyos, y A. Saldarriaga-Cardona. 2013. Evaluación in vitro de fungicidas comerciales para el control de Colletotrichum spp., en mora de castilla. Corpoica Cien. Tecnol. Agropecu. 14(1):67-75.

González-Vega, M.E. 2012. El ñame (Dioscorea spp.). Características, usos y valor medicinal. Aspectos de importancia en el desarrollo de su cultivo. Cul. Trop. 33(4):5-15.

Gutiérrez-Alonso, O., y J.G. Gutiérrez-Alonso. 2003. Evaluación de resistencia a benomil, thiabendazol y azoxystrobin para el control de antracnosis [Colletotrichum gloeosporioides (Penz.) Penz. and Sacc.] en frutos de guayaba (Psidium guajava L.) en postcosecha. Rev. Mex. Fitopatol. 21:228-232.

Gutiérrez-Alonso, J.G., O. Gutiérrez-Alonso, D. Nieto-Ángel, D. Téliz-Ortiz, E. Zavaleta-Mejía, F. Delgadillo-Sánchez, y H. Vaquera-Huerta. 2003. Resistencia a benomil y tiabendazol en aislamientos de Colletotrichum gloeosporioides (Penz.) Penz. y Sacc. obtenidos de mango (Mangifera indica L.) en cinco regiones de México. Rev. Mex. Fitopatol. 21:260-266.

Heaney, S.P., A.A. Hall, S.A. Davies, and G. Olaya. 2000. Resistance to fungicides in the Qol-STAR cross-resistance group: current perspectives. In: British Crop Protection Council, editor, Proceedings of the BCPC Conference: Pests and diseases. Vol. 2. British Crop Protection Council, Brighton, GBR. p. 755-762.

Hu, M.J., A. Grabke, M.E. Dowling, H.J. Holstein, and G. Schnabel. 2015. Resistance in Colletotrichum siamense from peach and blueberry to thiophanate-methyl and azoxystrobin. Plant Dis. 99:806-814. doi:10.1094/PDIS-10-14-1077-RE

IMN (Instituto Meteorológico Nacional). 2008. Atlas climatológico. https://www.imn.ac.cr/atlas-climatologico (consultado 28 jul. 2016).

INEC (Instituto Nacional de Estadística y Censos). 2015. VI censo nacional agropecuario: resultados generales. MAG, San José, CRI. http://www.mag.go.cr/bibliotecavirtual/U40-10581.pdf (consultado 31 ago. 2017).

InfoStat. 2011. InfoStat versión 2011. Grupo InfoStat, FCA, Universidad Nacional de Córdoba, ARG. http://www.infostat.com. ar (consultado 06 jul. 2016).

Ma, Z., and T.J. Michailides. 2005. Advances in understanding molecular mechanisms of fungicide resistance and molecular detection of resistant genotypes in phytopathogenic fungi. Crop Prot. 24:853-863. doi: 10.1016/j.cropro.2005.01 .011

Medaglia, C. 2012. El mercado de raíces y tubérculos en la Unión Europea. PROCOMER, CRI. http://servicios.procomer.go.cr/ aplicacion/civ/documentos/Estudio_Tuberculos_UE.pdf (consultado 06 jul. 2016).

Méndez, Y.P., J.L. Palencia, K.P. Hernández, E.J. Hernández, y J.D. Beltrán. 2013. Reacción de genotipos de ñame (Dioscorea spp) a la antracnosis (Colletotrichum gloeosporioides). Temas Agrarios 18(1):34-40. doi:10.21897/rta.v18i1.707

Pérez-León, G., R. Castillo-Matamoros, L. Chavarría-Pérez, A. Brenes-Angulo, y L. Gómez-Alpízar. 2015. Combate químico de la antracnosis de Sansevieria trifasciata var. Hahnii en un sistema de hojas separadas. Agron. Mesoam. 26:305-313. doi:10.15517/am.v26i2.19323 ISSN 2215-3608 doi:10.15517/am.v30i2.32653 
PROCOMER (Promotora de Comercio Exterior de Costa Rica). 2017. Portal estadístico de comercio exterior. PROCOMER, CRI. http://sistemas.procomer.go.cr/estadisticas/inicio.aspx (consultado 17 ene. 2018).

Raj, M., V. Hedge, M.L. Jeeva, M. Senthil, V.S. Nath, P. Vidyadharan, and P.V. Archana. 2013. Molecular diagnosis of Colletotrichum gloeosporioides causing anthracnose/dieback disease in greater yam (Dioscorea alata L.). Arch. Phytopathol. Plant Prot. 46:927-936. doi: 10.1080/03235408.2012.755336

Salazar, E., R. Hernández, A. Tapia, y L. Gómez-Alpízar. 2012. Identificación molecular del hongo Colletotrichum spp., aislado de banano (Musa spp) de altura en la zona de Turrialba y determinación de su sensibilidad a fungicidas poscosecha. Agron. Costarricense 36(1):53-68. doi:10.15517/rac.v36i1 9964

Sánchez, E. 2017. Identificación de patógenos asociados a antracnosis (Colletotrichum sp.) en el cultivo de ñame (Dioscorea alata) en la región Huetar Caribe de Costa Rica. Tesis Bach., Instituto Tecnológico de Costa Rica, Santa Clara, CRI.

Sierotzki, H., R. Frey, J. Wullschleger, S. Palermo, S. Karlin, J. Godwin, and U. Gisi. 2007. Cytochrome b gene sequence and structure of Pyrenophora teres and P. tritici-repentis and implications for QoI resistance. Pest Manag. Sci. 63:225-233. doi: $10.1002 / p s .1330$

Suwannarat, S., S. Steinkellner, P. Songkumarn, and S. Sangchote. 2017. Diversity of Colletotrichum spp. isolated from chili pepper fruit exhibiting symptoms of anthracnose in Thailand. Mycol. Prog. 16:677-686. doi:10.1007/s11557-017-1304-2

Tavares, G.M., e P.E. de-Souza. 2005. Efeito de fungicidas no controle in vitro de Colletotrichum gloeosporioides, agente etiológico da antracnose do mamoeiro (Carica papaya L.). Ciênc. Agrotecnol. 29:52-59. doi:10.1590/S14137054005000100006

Torres-Calzada, C., R. Tapia-Tussell, I. Higuera-Ciapara, R. Martin-Mex, A. Nexticapan-Garcez, and D. Perez-Brito. 2015. Sensitivity of Colletotrichum truncatum to four fungicides and characterization of thiabendazole-resistant isolates. Plant Dis. 99:1590-1595. doi:10.1094/PDIS-11-14-1183-RE

Trinidad-Cruz, J., E. Quiñones-Aguilar, J. Qui-Zapata, J. Rodríguez-Domínguez, L. López-Pérez, y G. Rincón-Enríquez. 2013. Patogenicidad de Fusarium oxysporum en Agave cupreata. Rev. Mex. Fitopatol. 31:S90.

White, T.J., T. Bruns., S. Lee, and J. Taylor. 1990. Amplification and direct sequencing of fungal ribosomal RNA genes for phylogenetics. In: M. Innis et al., editors, PCR protocols: A guide to methods and applications. Academic Press, San Diego, CA, USA. p. 315-322.

Weir, B.S., P.R. Johnston, and U. Damm, 2012. The Colletotrichum gloeosporioides species complex. Stud. Mycol. 73:115-180. doi: $10.3114 / \operatorname{sim} 0011$ 